\title{
Taylor term does not imply any nontrivial linear one-equality Maltsev condition
}

\author{
AleXANDR KAZDA
}

\begin{abstract}
It is known that any finite idempotent algebra that satisfies a nontrivial Maltsev condition must satisfy the linear one-equality Maltsev condition (a variant of the term discovered by M. Siggers and refined by K. Kearnes, P. Marković, and R. McKenzie):

$$
t(r, a, r, e) \approx t(a, r, e, a) .
$$

We show that if we drop the finiteness assumption, the $k$-ary weak near unanimity equations imply only trivial linear one-equality Maltsev conditions for every $k \geq 3$. From this it follows that there is no nontrivial linear one-equality condition that would hold in all idempotent algebras having Taylor terms.

Miroslav Olšák has recently shown that there is a weakest nontrivial strong Maltsev condition for idempotent algebras. Olšák has found several such (mutually equivalent) conditions consisting of two or more equations. Our result shows that Olšák's equation systems can't be compressed into just one equation.
\end{abstract}

\section{Introduction}

In this note we show that for every $k \geq 3$ the free algebra with a $k$-ary weak near unanimity term does not satisfy any nontrivial linear one-equality Maltsev condition. This is in contrast to the finite case where having a Taylor term means that the algebra in question has the Siggers term [7. The original Siggers term is equivalent [4, Theorem 2.2.] to the single equation form (the mnemonics for names of variables is due to Ryan O'Donnel):

$$
t(r, a, r, e) \approx t(a, r, e, a) .
$$

Miroslav Olšák has recently shown that having a Taylor term is a strong Maltsev condition even for infinite (idempotent) algebras [6]. Olšák's shortest condition consists of two linear identities and it would be natural to ask if one can't do better and use only one equation. This paper shows that such an improvement is impossible.

\section{Preliminaries}

An algebra $\mathbf{A}$ consists of a base set $A$ on which acts a set of basic operations of $\mathbf{A}$. An operation is a mapping $f: A^{n} \rightarrow A$ where $n \in \mathbb{N}$ is the arity of $f$.

This work was supported by European Research Council under the European Union's Seventh Framework Programme (FP7/2007-2013)/ERC grant agreement no 616160, the PRIMUS/SCI/12 and UNCE/SCI/022 projects of the Charles University. 
The clone of operations of $\mathbf{A}$ is the smallest set of operations that contains all basic operations of $\mathbf{A}$ as well as projections (that is, operations of the form $\left.\pi_{i}^{k}\left(x_{1}, \ldots, x_{k}\right)=x_{i}\right)$ and is closed under composing operations. An algebra is idempotent if for any operation $t$ of $\mathbf{A}$ and any $a \in A$ we have $t(a, \ldots, a)=a$. It is an easy exercise to verify that for $\mathbf{A}$ to be idempotent it suffices that just the basic operations of $\mathbf{A}$ are all idempotent.

A term of $\mathbf{A}$ is a (syntactically correct) composition of basic operation symbols of $\mathbf{A}$ and variables. An equational identity, or equation, is a statement of the form " $u \approx v "$ where $u$ and $v$ are terms and the symbol " $\approx$ " stands for "the left hand side equals the right hand side after any assignment of members of $A$ to variables." An example of an identity is $t(x, \ldots, x) \approx x$ which says that the operation $t$ is idempotent.

A variety is a class of algebras sharing the same signature (the same basic operation symbols and arities of basic operations) that is closed under taking subalgebras, products and homomorphic images, or equivalently(by Birkhoff's theorem [3. Theorem 4.41]) a class of algebras defined by a system of equational identities. The set of all identities that holds in a variety is called the equational theory of the variety.

A strong Maltsev condition is a finite list of identities involving some operation symbols. An algebra A satisfies a strong Maltsev condition $M$ if for each $k$-ary operation symbol in $M$ one can choose a $k$-ary operation of $\mathbf{A}$ so that when we replace the symbols of $M$ by operations of $\mathbf{A}$, we get a system of equations that are all true in $\mathbf{A}$. A variety satisfies the condition $M$ if all algebras in the variety satisfy $M$. Since we consider only strong Maltsev conditions in this paper, we will omit the adjective "strong" for brevity.

A Maltsev condition is trivial if it is satisfied by the algebra $\mathbf{P}$ on two elements 0 and 1 whose set of operations consists of projections only. An example of a trivial strong Maltsev condition is $t(t(x, y, z), y, z) \approx t(x, x, z)$; one can satisfy this condition by choosing $t$ to be the third projection (i.e. $t(a, b, c)=c$ for all $a, b, c)$.

A Maltsev condition is called linear if its identities don't involve compositions, i.e. all identities have the form $t(\bar{x}) \approx s(\bar{y})$ or $t(\bar{x}) \approx z$ or $z \approx r$ where $\bar{x}, \bar{y}$ are tuples of variables, $t, s$ are (possibly equal) operation symbols, and $z, r$ are variables (we include the third case for completeness only; only a trivial algebra can satisfy an identity of the form $z \approx r$ where $z, r$ are distinct variables).

Having a $k$-ary weak near unanimity operation ( $k$-wnu) for a fixed $k \in$ $\{2,3,4, \ldots\}$ is a Maltsev condition that consists of the following $k$ linear identities for the $k$-ary operation symbol $w$ :

$$
\begin{aligned}
w(x, x, x, \ldots, x, x) & \approx x \\
w(y, x, x, \ldots, x, x) & \approx w(x, y, x, \ldots, x, x) \approx w(x, x, y, \ldots, x, x) \approx \ldots \\
\cdots & \approx w(x, x, x, \ldots, y, x) \approx w(x, x, x, \ldots, x, y) .
\end{aligned}
$$


Having a Taylor operation (term) refers to having an operation $t$ satisfying any linear Maltsev condition of the form

$$
\begin{aligned}
t(x, \ldots, x) & \approx x \\
t(x, ?, ?, \ldots, ?) & \approx t(y, ?, ?, \ldots, ?) \\
t(?, x, ?, \ldots, ?) & \approx t(?, y, ?, \ldots, ?) \\
& \vdots \\
t(?, ?, ?, \ldots, x) & \approx t(?, ?, ?, \ldots, y),
\end{aligned}
$$

where $x, y$ are variables and the question marks stand for some choice of $x$ 's and $y$ 's.

It is immediate to see that any operation that is a $k$-wnu is also a Taylor term (but not the other way around). Finite idempotent algebras with Taylor terms are well understood as the following theorem shows.

Theorem 1 (Combining 8], [5], 4], and 2]). Let $\mathbf{A}$ be a finite idempotent algebra. Then the following are equivalent:

(1) A satisfies a nontrivial Maltsev condition,

(2) A has a Taylor term,

(3) A has a $k$-wnu for some $k \in \mathbb{N}$,

(4) A has a $k$-ary cyclic term for some $k \in \mathbb{N}$, where a cyclic term satisfies the equation

$$
c\left(x_{1}, x_{2}, \ldots, x_{k}\right) \approx c\left(x_{k}, x_{1}, \ldots, x_{k-1}\right) .
$$

(5) A satisfies the Maltsev condition (known as a Siggers term)

$$
t(r, a, r, e) \approx t(a, r, e, a)
$$

where $a, e, r$ are variables.

Note that the cyclic and Siggers term conditions, unlike the other equivalent conditions involve only one identity (plus idempotency, which we assume from the start). We will abbreviate single linear equality Maltsev condition as $S L E M C$. Siggers term and cyclic term conditions are examples of nontrivial SLEMCs, while the 3 -wnu condition is not a SLEMC.

Our work stems from an attempt to generalize Theorem 1 to infinite idempotent algebras. We will show that there is no analogue of the last two points, i.e. that having a $k$-wnu for $k \geq 3$ does not imply a nontrivial SLEMC. (For $k=2$, we have the SLEMC $w(x, y) \approx w(y, x)$.)

\section{3-wnu implies only trivial SLEMCs}

In this section we show in detail that having a 3-wnu term does not imply any nontrivial SLEMC. The general case of having a $k$-wnu differs from the 3 -wnu situation only by a slightly more complicated notation. This is why 
we first give the proof for 3 -wnu and then, in the next section, we sketch the argument for the general case without going into details.

Theorem 2. Let $V$ be the variety of algebras with one ternary basic operation $w$ and with the equational theory generated by the 3-wnu identities

$$
\begin{aligned}
& w(x, x, x) \approx x \\
& w(x, x, y) \approx w(x, y, x) \approx w(y, x, x) .
\end{aligned}
$$

This variety (which is idempotent and has a 3-wnu term) does not satisfy any nontrivial SLEMC.

The proof of this theorem will occupy the rest of this section. From the equations, we can see that $V$ is idempotent and that $w$ is a 3 -wnu operation, so the only nonobvious statement is that $V$ does not satisfy any nontrivial SLEMC.

Since $V$ contains algebras on more than one element (for example $\{0,1\}$ with $w(x, y, z)=x+y+z(\bmod 2))$, any candidate for a nontrivial SLEMC has to have a rather specific shape:

Observation 3. Let $\mathbf{A}$ be an idempotent algebra on at least two elements. If A satisfies a nontrivial SLEMC $M$, then $M$ has the form

$$
t\left(x_{1}, \ldots, x_{m}\right) \approx t\left(y_{1}, \ldots, y_{m}\right)
$$

where $t$ is an operation symbol and $x_{1}, \ldots, x_{m}, y_{1}, \ldots, y_{m}$ are variable symbols such that $x_{i} \neq y_{i}$ for $i=1,2, \ldots, m$.

Proof. Assume that A satisfies a nontrivial SLEMC $M$ of the form

$$
r\left(x_{1}, \ldots, x_{m}\right) \approx s\left(y_{1}, \ldots, y_{k}\right)
$$

where $r, s$ are two different operation symbols. Since the condition $M$ is supposed to be nontrivial, the variable sets $\left\{x_{1}, \ldots, x_{m}\right\}$ and $\left\{y_{1}, \ldots, y_{k}\right\}$ must be disjoint (for had we $x_{i}=y_{j}$ then we could satisfy $M$ by taking $r$ and $s$ to be the projections to the $i$-th and $j$-th coordinates, respectively). Therefore, the SLEMC $M$ implies

$$
r(x, \ldots, x) \approx s(y, \ldots, y)
$$

where $x, y$ are distinct variable symbols. Since $\mathbf{A}$ is idempotent, the operations of $\mathbf{A}$ realizing $r$ and $s$ are idempotent and $\mathbf{A}$ satisfies $x \approx y$, meaning $|A|=1$.

A similar argument rules out the SLEMC

$$
t\left(x_{1}, \ldots, x_{m}\right) \approx y .
$$

This leaves only the possibility

$$
t\left(x_{1}, \ldots, x_{m}\right) \approx t\left(y_{1}, \ldots, y_{m}\right),
$$

where $x_{i} \neq y_{i}$ for all $i$ (were $x_{i}=y_{i}$, we could satisfy $M$ by taking $t$ to be the $m$-ary projection to the $i$-th coordinate). 
We will now construct an algera in $V$ that satisfies no nontrivial SLEMC. Two comments before we begin: First, we are actually going to construct the free countably generated algebra in $V$. Second, we note for readers familiar with term rewrite systems (see eg. 1]) that we are implicitly studying the term rewrite system with the rules $w(x, x, x) \rightarrow x, w(y, x, x), w(x, y, x), w(x, x, y) \rightarrow$ $u(x, y)$ where $u(x, y)$ is a new symbol that stands for $w(y, x, x)$. We opted to not use the machinery of term rewriting because an elementary argument is reasonably short and prepares us for calculations with normal forms later on.

Let $X$ be a countable set of variable symbols. Let $T$ be the set of all possible terms we can get using $X$ and a single ternary operation symbol $w$ (so for example $w(y, w(x, y, z), y) \in T$ ).

We define the set $A$ of "normal forms" of terms of $T$ modulo the 3 -wnu identities as follows: A term $t$ lies in $A$ if either $t$ is a variable from $X$, or $t=w\left(a_{1}, a_{2}, a_{3}\right)$ where $a_{1}, a_{2}, a_{3} \in A$ and we have $a_{1} \neq a_{2}, a_{3}$ (for example, $w(w(x, y, z), y, y)$ lies in $A$, but $w(y, w(x, y, z), y)$ does not).

Let $t \in T$ be a term. It is easy to prove by induction on the number of occurrences of $w$ in $t$ that we can use the 3 -wnu identitites to rewrite $t$ to a term $t^{\prime} \in A$ such that $t \approx t^{\prime}$ in $V$ (in fact, the term $t^{\prime}$ is unique for a given $t$; we omit the proof of this as we will not need it).

Consider the algebra $\mathbf{A}=\left(A, w^{\mathbf{A}}\right)$ with the operation $w^{\mathbf{A}}$ defined as follows:

(1) If $a_{1}, a_{2}, a_{3} \in A$ are pairwise different then we let $w^{\mathbf{A}}\left(a_{1}, a_{2}, a_{3}\right)=$ $w\left(a_{1}, a_{2}, a_{3}\right)$,

(2) if $a, b \in A$ are different then we let all three of $w^{\mathbf{A}}(a, a, b), w^{\mathbf{A}}(a, b, a)$, $w^{\mathbf{A}}(b, a, a)$ to be equal to $w(b, a, a)$, and

(3) if $a \in A$, then $w^{\mathbf{A}}(a, a, a)=a$.

It is easy to verify that $\mathbf{A}$ is closed under $w^{\mathbf{A}}$. Observe also that the operation $w^{\mathbf{A}}$ is a 3 -wnu operation, so $\mathbf{A} \in V$.

Note that in many cases we have $w^{\mathbf{A}}(a, b, c)=w(a, b, c)$, but this is not always true. This is why we distinguish between $w^{\mathbf{A}}$ (operation symbol of $\mathbf{A}$ ) and $w$ (formal symbol used to describe terms of $V$ ).

Since $\mathbf{A} \in V$, to prove Theorem 2 it is enough to show that $\mathbf{A}$ satisfies only trivial SLEMCs. To that end let $R$ be the subalgebra of $\mathbf{A}^{2}$ generated by $\{(x, y): x, y \in X, x \neq y\}$. The following observation shows that to prove Theorem 2, it is enough to show that $R$ does not intersect the diagonal.

Observation 4. If the variety $V$ satisfies a nontrivial SLEMC, then the relation $R$ defined above intersects the diagonal of $A^{2}$ (in other words, there is an $r \in A$ such that $(r, r) \in R)$.

Proof. Assume that $V$ satisfies the SLEMC

$$
t\left(y_{1}, \ldots, y_{m}\right) \approx t\left(z_{1}, \ldots, z_{m}\right)
$$

where $y_{1}, \ldots, y_{m}, z_{1}, \ldots, z_{m}$ are variables (without loss of generality) taken from the set $X$. Let us denote by $t^{\mathbf{A}}$ the term of $\mathbf{A}$ we obtain from $t$ by 
replacing each symbol $w$ by $w^{\mathbf{A}}$. In $\mathbf{A}$ we thus have the equality (note that $y_{i}$ 's and $z_{i}$ 's are members of $A$ here, not variable symbols).

$$
t^{\mathbf{A}}\left(y_{1}, \ldots, y_{m}\right)=t^{\mathbf{A}}\left(z_{1}, \ldots, z_{m}\right)=r .
$$

for some $r \in A$.

We have $\left(y_{i}, z_{i}\right) \in R$ for all $i$ and so applying the operation $t^{\mathbf{A}}$ to pairs $\left(y_{1}, z_{1}\right), \ldots,\left(y_{m}, z_{m}\right) \in R$ gets us $(r, r) \in R$.

While we would like to show that $R$ does not intersect the diagonal, idempotency prevents us from comfortably doing a proof by induction on term complexity on $R$ itself. This is why we take a detour through subterms.

Definition 5. We define the relation "to be a subterm" on the set $A$, denoted by $\preceq$, as the reflexive and transitive closure of the set

$$
Q=\{(a, b): a, b \in A, \exists c, d, e \in A, b=w(c, d, e), a \in\{c, d, e\}\} .
$$

Informally, $a \preceq b$ if in the term $b$ we can find a subterm that is identical to $a$. Note that $\preceq$ is defined using the (syntactic) symbol $w$. However, it turns out that $\preceq$ behaves well with respect to the operation $w^{\mathbf{A}}$, too:

Lemma 6. The following holds for $\preceq$ :

(a) If $x, y$ are distinct members of $X$ (i.e. variables), then $x \npreceq y$.

(b) For all $b, c, d \in A$, we have $b \preceq w^{\mathbf{A}}(b, c, d), w^{\mathbf{A}}(d, b, c), w^{\mathbf{A}}(c, d, b)$.

(c) For all $a, b, c, d \in A$ such that $a \preceq b$ we have $a \preceq w^{\mathbf{A}}(b, c, d), w^{\mathbf{A}}(d, b, c)$, $w^{\mathbf{A}}(c, d, b)$.

(d) If $a, b, c, d \in A$ are such that $a \preceq w^{\mathbf{A}}(b, c, d)$ and $a \npreceq b, c, d$, then $a=$ $w^{\mathbf{A}}(b, c, d)$.

Proof. (a) Since $x \neq y$, the only way we could have had $x \preceq y$ would be if there was a chain of $k \geq 2$ terms $x=t_{1}, t_{2}, \ldots, t_{k}=y$ such that $\left(t_{i}, t_{i+1}\right) \in Q$ for $i=1, \ldots, k-1$ (where $Q$ is the set from the definition of $\preceq$ ). From this we get that for all $i=1, \ldots, k-1$ we have $t_{i+1}=w\left(p_{i}, q_{i}, r_{i}\right)$ where $p_{i}, q_{i}, r_{i}$ are members of $A$ and $t_{i}$ appears at least once in $\left(p_{i}, q_{i}, r_{i}\right)$. By induction on $i$, it follows that each $t_{i}$ must have at least $i-1$ occurrences of the symbol $w$, so $t_{k}$ contains at least one symbol $w$. But the term $t_{k}=y$ has no $w$ in it, a contradiction.

(b) We will show $b \preceq w^{\mathbf{A}}(b, c, d)$; the other two subterm relationships are similar.

Unless $b=c=d$, we have $w^{\mathbf{A}}(b, c, d) \in\{w(b, c, d), w(d, b, c), w(c, d, b)\}$ and $b$ is a subterm of each of the terms on the right side, so we are done. In the case $b=c=d$, we get $w^{\mathbf{A}}(b, c, d)=b$ and $b \preceq b$ follows from the reflexivity of $\preceq$.

(c) This follows from the transitivity of $\preceq$ and the previous point: We have $a \preceq b \preceq w^{\mathbf{A}}(b, c, d), w^{\mathbf{A}}(d, b, c), w^{\mathbf{A}}(c, d, b)$. 
(d) Were $b, c, d$ all equal, we would have $a \preceq w^{\mathbf{A}}(b, b, b)=b$, a contradiction. with $a \npreceq b$. Therefore, without loss of generality $w^{\mathbf{A}}(b, c, d)=w(b, c, d)$ (we can reorder $b, c, d$ ).

Assume for a contradiction that $a \neq w^{\mathbf{A}}(b, c, d)=w(b, c, d)$. Since the relation $a \preceq w(b, c, d)$ is not a consequence of reflexivity, there is a $k \geq 2$ and a chain $a=t_{1} \preceq t_{2} \preceq \cdots \preceq t_{k-1} \preceq t_{k}=w(b, c, d)$ witnessing $a \preceq w(b, c, d)$ with $\left(t_{i}, t_{i+1}\right) \in Q$ for all $i$. But then $a \preceq t_{k-1}$ and $t_{k-1}$ needs to be one of $b, c, d$ by the definition of $Q$, a contradiction with $a \npreceq b, c, d$.

Let now $S$ be the following relation on $A$ :

$$
S=\left\{(a, b) \in A^{2}: a \npreceq b \wedge b \npreceq a\right\} .
$$

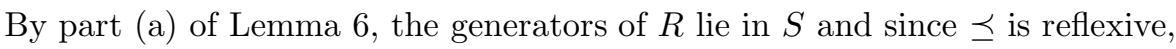
$S$ does not intersect the diagonal.

Lemma 7. The relation $S$ is a subuniverse of $\mathbf{A}^{2}$.

Proof. Let us take $\left(a_{1}, b_{1}\right),\left(a_{2}, b_{2}\right),\left(a_{3}, b_{3}\right) \in S$ such that (without loss of generality) $w^{\mathbf{A}}\left(a_{1}, a_{2}, a_{3}\right) \preceq w^{\mathbf{A}}\left(b_{1}, b_{2}, b_{3}\right)$.

We consider several cases:

(a) Assume that $w^{\mathbf{A}}\left(a_{1}, a_{2}, a_{3}\right) \neq w^{\mathbf{A}}\left(b_{1}, b_{2}, b_{3}\right)$. Then by part (d) of Lemma 6 , $w^{\mathbf{A}}\left(a_{1}, a_{2}, a_{3}\right)$ (which plays the role of $a$ in the Lemma) needs to be a subterm of one of $b_{1}, b_{2}, b_{3}$. Without loss of generality assume $w^{\mathbf{A}}\left(a_{1}, a_{2}, a_{3}\right) \preceq$ $b_{1}$. But $a_{1} \preceq w^{\mathbf{A}}\left(a_{1}, a_{2}, a_{3}\right)$ by part (IC) of Lemma 6 . We have $a_{1} \preceq$ $w^{\mathbf{A}}\left(a_{1}, a_{2}, a_{2}\right) \preceq b_{1}$, which is a contradiction with $\left(a_{1}, b_{1}\right) \in S$.

(b) Assume that $w^{\mathbf{A}}\left(a_{1}, a_{2}, a_{3}\right)=w^{\mathbf{A}}\left(b_{1}, b_{2}, b_{3}\right)$ and $a_{1}=a_{2}=a_{3}$. Then $b_{1} \preceq w^{\mathbf{A}}\left(b_{1}, b_{2}, b_{3}\right)=w^{\mathbf{A}}\left(a_{1}, a_{1}, a_{1}\right)=a_{1}$ (where the subterm relationship follows again by part (ㄷ) of Lemma (6) and therefore $b_{1} \preceq a_{1}$.

The same argument takes care of the case $b_{1}=b_{2}=b_{3}$.

(c) Assume that $w^{\mathbf{A}}\left(a_{1}, a_{2}, a_{3}\right)=w^{\mathbf{A}}\left(b_{1}, b_{2}, b_{3}\right)$ and $\left\{a_{1}, a_{2}, a_{3}\right\}=\{c, d\}$ with $d$ appearing twice, i.e. $w^{\mathbf{A}}\left(a_{1}, a_{2}, a_{3}\right)=w(c, d, d)=w^{\mathbf{A}}\left(b_{1}, b_{2}, b_{3}\right)$

Since $b_{1}, b_{2}, b_{3}$ are not all equal, by the definition of $w^{\mathbf{A}}$ we must have $\left\{b_{1}, b_{2}, b_{3}\right\}=\{c, d\}$ with $d$ appearing twice. Since we have three pairs $\left(a_{1}, b_{1}\right),\left(a_{2}, b_{2}\right),\left(a_{3}, b_{3}\right)$ and only two appearances of $c$ (one for $a$ 's, one for $b$ 's), it follows that there exists an $i$ such that $a_{i}=d=b_{i}$. However, $(d, d) \notin S$, a contradiction.

(d) Assume that $w^{\mathbf{A}}\left(a_{1}, a_{2}, a_{3}\right)=w^{\mathbf{A}}\left(b_{1}, b_{2}, b_{3}\right)$ and $a_{1}, a_{2}, a_{3}$ are pairwise different, i,e. $w\left(a_{1}, a_{2}, a_{3}\right)=w^{\mathbf{A}}\left(b_{1}, b_{2}, b_{3}\right)$. Since $b_{1}, b_{2}, b_{3}$ are not all equal, the only way to get equality here is to have $a_{i}=b_{i}$ for all $i=1,2,3$, a contradiction with $\left(a_{i}, b_{i}\right) \in S$.

Proof of Theorem Q 2 . By Lemma 7, we get that $S$ is a subuniverse of $\mathbf{A}^{2}$ that contains all generators of $R$ and thus $R \subseteq S$. As we have seen above, $S$ is 
disjoint from the diagonal, so $R$ must be disjoint from the diagonal. Therefore, by Observation 4 , the variety $V$ can't satisfy a nontrivial SLEMC.

\section{4. $k$-wnu implies only trivial SLEMCs}

Theorem 8. For any $k \geq 3$ the $k$-wnu identities don't imply a nontrivial SLEMC.

Proof. The proof is very similar to the proof of Theorem 2 so we only sketch the main points here.

We take $V$ to be the variety defined by the $k$-wnu equations for a $k$-wnu operation $w, X$ a countable set of variables and $A$ the smallest set of terms made from $X$ and $w$ such that $X \subseteq A$ and $w\left(a_{1}, \ldots, a_{k}\right) \in A$ if and only if $a_{1}, \ldots, a_{k} \in A$ and there are at least two distinct indices $i, j$ such that $a_{1} \neq a_{i}, a_{j}$.

Again, we consider the algebra $\mathbf{A}=\left(A, w^{\mathbf{A}}\right) \in V$ with $w^{\mathbf{A}}\left(a_{1}, \ldots, a_{k}\right)$ defined as

$$
w^{\mathbf{A}}\left(a_{1}, \ldots, a_{k}\right)= \begin{cases}a_{1} & a_{1}=a_{2}=\cdots=a_{k} \\ w(c, d, d, \ldots, d) & \exists i \in\{1, \ldots, k\}, a_{i}=c \\ & a_{1}=a_{2}=\cdots=a_{i-1}=a_{i+1}=\cdots \\ & \cdots=a_{k}=d \neq c \\ w\left(a_{1}, a_{2}, \ldots, a_{k}\right) & \text { otherwise. }\end{cases}
$$

The relation $R$ is again generated in $\mathbf{A}^{2}$ by $\{(x, y) \in X: x \neq y\}$, while the subterm relation $\preceq$ is defined as the reflexive and transitive closure of

$$
\left\{(a, b): a, b \in A, \exists c_{1}, c_{2}, \ldots, c_{k} \in A, b=w\left(c_{1}, \ldots, c_{k}\right), a \in\left\{c_{1}, \ldots, c_{k}\right\}\right\} .
$$

As before, we show that

$$
S=\left\{(a, b) \in A^{2}: a \npreceq b \wedge b \npreceq a\right\}
$$

is $\mathbf{A}$-invariant and thus prove that $R$ does not intersect the diagonal which implies that $V$ satisfies no nontrivial SLEMC.

\section{REFERENCES}

[1] Franz Baader and Tobias Nipkow. Term Rewriting and All That. Cambridge University Press, New York, 1999.

[2] Libor Barto and Marcin Kozik. Absorbing subalgebras, cyclic terms, and the constraint satisfaction problem. Logical Methods in Computer Science, 8(1), 2012.

[3] Clifford Bergman. Universal Algebra: Fundamentals and Selected Topics. Chapman \& Hall/CRC Press, Boca Raton and New York and Abingdon, 1st edition, 2011.

[4] Keith Kearnes, Petar Marković, and Ralph McKenzie. Optimal strong Mal'cev conditions for omitting type 1 in locally finite varieties. Algebra universalis, 72(1):91-100, 2014.

[5] Miklós Maróti and Ralph McKenzie. Existence theorems for weakly symmetric operations. Algebra Universalis, 59:463-489, 2008.

[6] Miroslav Olšák. The weakest nontrivial idempotent equations. Bulletin of the London Mathematical Society, 49(6):1028-1047, 2017. 
[7] Mark H. Siggers. A strong Mal'cev condition for locally finite varieties omitting the unary type. Algebra universalis, 64(1):15-20, 2010.

[8] Walter Taylor. Varieties obeying homotopy laws. Canadian Journal of Mathematics, 29:498-527, 1977.

AlexANDR KAZDA

Department of Algebra, Charles University, Sokolovská 83, 186 75, Prague, Czechia, ORCID:0000-0002-7338-037X

e-mail: alex.kazda@gmail.com 